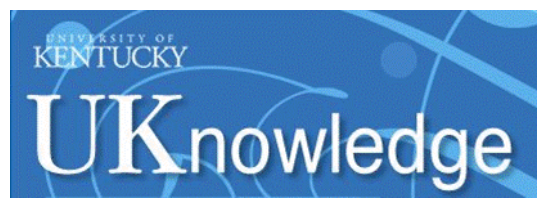

University of Kentucky

UKnowledge

$11-4-2016$

\title{
Sign-Problem-Free Monte Carlo Simulation of Certain Frustrated Quantum Magnets
}

\author{
Fabien Alet \\ Université de Toulouse, France \\ Kedar Damle \\ Tata Institute of Fundamental Research, India \\ Sumiran Pujari \\ University of Kentucky, sumiran.pujari@uky.edu
}

Follow this and additional works at: https://uknowledge.uky.edu/physastron_facpub

Part of the Physics Commons

Right click to open a feedback form in a new tab to let us know how this document benefits you.

\section{Repository Citation}

Alet, Fabien; Damle, Kedar; and Pujari, Sumiran, "Sign-Problem-Free Monte Carlo Simulation of Certain Frustrated Quantum Magnets" (2016). Physics and Astronomy Faculty Publications. 405.

https://uknowledge.uky.edu/physastron_facpub/405

This Article is brought to you for free and open access by the Physics and Astronomy at UKnowledge. It has been accepted for inclusion in Physics and Astronomy Faculty Publications by an authorized administrator of UKnowledge. For more information, please contact UKnowledge@lsv.uky.edu. 


\title{
Sign-Problem-Free Monte Carlo Simulation of Certain Frustrated Quantum Magnets
}

\author{
Digital Object Identifier (DOI) \\ https://doi.org/10.1103/PhysRevLett.117.197203 \\ Notes/Citation Information \\ Published in Physical Review Letters, v. 117, issue 19, 197293, p. 1-7. \\ (C) 2016 American Physical Society
}

The copyright holder has granted the permission for posting the article here. 


\title{
Sign-Problem-Free Monte Carlo Simulation of Certain Frustrated Quantum Magnets
}

\author{
Fabien Alet, ${ }^{1}$ Kedar Damle, ${ }^{2}$ and Sumiran Pujari ${ }^{3}$ \\ ${ }^{1}$ Laboratoire de Physique Théorique, IRSAMC, Université de Toulouse, CNRS, 31062 Toulouse, France \\ ${ }^{2}$ Department of Theoretical Physics, Tata Institute of Fundamental Research, Mumbai 400 005, India \\ ${ }^{3}$ Department of Physics and Astronomy, University of Kentucky, Lexington, Kentucky 40506-0055, USA \\ (Received 21 January 2016; revised manuscript received 27 August 2016; published 4 November 2016)
}

We introduce a quantum Monte Carlo (QMC) method for efficient sign-problem-free simulations of a broad class of frustrated $S=1 / 2$ antiferromagnets using the basis of spin eigenstates of clusters to avoid the severe sign problem faced by other QMC methods. We demonstrate the utility of the method in several cases with competing exchange interactions and flag important limitations as well as possible extensions of the method.

DOI: 10.1103/PhysRevLett.117.197203

Quantum Monte Carlo (QMC) simulations compute equilibrium properties of a many-body system by importance sampling of the canonical partition function $Z=$ $\operatorname{Tr} \exp (-H / T)$, where $H$ is the many-body Hamiltonian and $T$ is the temperature [1-3]. They have emerged as a major tool for the study of lattice Hamiltonians that either model low- $T$ thermodynamic properties of interesting strongly correlated materials [4,5] or provide concrete realizations of novel phases in such condensed matter systems [6,7]. However, models of geometrically frustrated magnets [5], in which antiferromagnetic interactions compete with each other due to the geometry of the exchange pathways, have typically remained beyond the reach of QMC methods. This is due to the presence of a sign problem, whereby the weights assigned to individual Monte Carlo configurations are no longer strictly positive in the commonly used basis of eigenstates of $S_{\vec{r}}^{z}$, the $z$ component of each spin. In such cases, the average sign decreases exponentially with the system size and inverse temperature, leading to unmanageably large statistical errors in the estimation of physical quantities. A similar sign problem crops up in diverse settings ranging from QCD to strongly correlated metals, and a general solution is considered unlikely [8].

Limited progress has been possible in a few cases, for instance, in anisotropic systems in which the frustration affects only $S_{\vec{r}}^{z}$ (thereby allowing sign-free simulation in the $z$ basis [9-17]) or when the sign problem of the full theory can be finessed at low $T$ by working with a low-energy effective Hamiltonian which has no sign problem [18-22]. For some models, sign-free simulations are possible by virtue of specific symmetries of the Hamiltonian [23-29]. In other strongly correlated systems with a full-fledged sign problem, progress has been made in some cases by developing improved estimators for computing physical observables [30-33]. In principle, one could also try to find an alternate basis in which all configurations have positive weights. However, this has been possible only in a few interesting cases [34,35], including some models of topologically ordered states of matter [36-38].

Synopsis.-In this Letter, we introduce a QMC method that works in the basis of spin eigenstates of clusters to simulate a large class of frustrated quantum magnets in a provably sign-free manner. We focus our discussion on systems in which the clusters in question are made up of two spin-1/2 moments $\left(\vec{S}_{\mathrm{I} r}\right.$ and $\left.\vec{S}_{\mathrm{II} r}\right)$ located on layers I and II at sites $r$ of a bipartite Bravais lattice in any spatial dimension (Fig. 1), with Hamiltonian

$$
\begin{aligned}
H_{\text {bilayer }}= & \sum_{\left\langle r_{a} r_{b}\right\rangle}\left(\mathcal{J}_{z} S_{\mathrm{I} r_{a}}^{z} S_{\mathrm{I} r_{b}}^{z}+\mathcal{J}_{\perp} \vec{S}_{\mathrm{I} r_{a}}^{\perp} \cdot \vec{S}_{\mathrm{I} r_{b}}^{\perp}+\mathrm{I} \leftrightarrow \mathrm{II}\right) \\
& +\sum_{\left\langle r_{a} r_{b}\right\rangle}\left(\mathcal{K}_{z} S_{\mathrm{I} r_{a}}^{z} S_{\mathrm{II} r_{b}}^{z}+\mathcal{K}_{\perp} \vec{S}_{\mathrm{I} r_{a}}^{\perp} \cdot \vec{S}_{\mathrm{II} r_{b}}^{\perp}+\mathrm{I} \leftrightarrow \mathrm{II}\right) \\
& +\sum_{r}\left(\mathcal{D}_{z} S_{\mathrm{I} r}^{z} S_{\mathrm{II} r}^{z}+\mathcal{D}_{\perp} \vec{S}_{\mathrm{I} r}^{\perp} \cdot \vec{S}_{\mathrm{II} r}^{\perp}\right),
\end{aligned}
$$

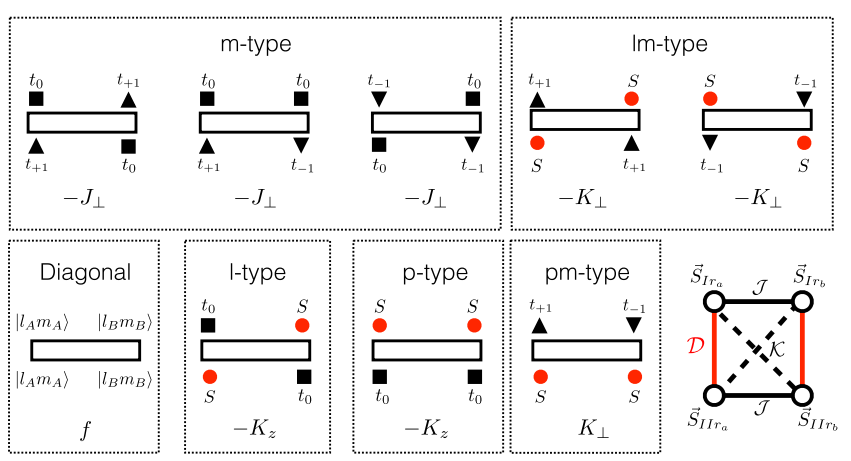

FIG. 1. Vertices that appear in the SSE operator string for $H_{\text {bilayer, }}$ with corresponding weights in the canonical cluster basis. All other valid vertices are obtained by symmetry operations that exchange left and right, or upper and lower, legs (keeping the weight fixed). The constant $C$ in the function $f\left(l_{A}, l_{B}, m_{A}, m_{B}\right)=C-J_{z} m_{A} m_{B}-$ $\zeta\left(\Delta_{z}-\Delta_{\perp}\right)\left(m_{A}^{2}+m_{B}^{2}\right)-\zeta \Delta_{\perp}\left[l_{A}\left(l_{A}+1\right)+l_{B}\left(l_{B}+1\right)\right]$ is chosen to ensure that $f \geq 0$. Bottom right: Lattice structure and exchange couplings of $H_{\text {bilayer }}$. Vertices and lattice structure for $H_{\text {mixed }}$ are detailed in Supplemental Material [56]. 
where the nearest-neighbor links of this Bravais lattice have been denoted by $\left\langle r_{a} r_{b}\right\rangle$ to emphasize its bipartite nature and $\vec{S}_{\mathrm{I} / \mathrm{II} r}^{\perp}$ represents the vector formed by the two transverse components ( $x$ and $y$ ) of these spins. Geometric frustration of the exchange interactions leads to a severe sign problem for other QMC methods whenever $\mathcal{D}_{\perp} \mathcal{K}_{\perp} \mathcal{J}_{\perp}>0$. Our central result is that such frustration leads to no sign problems in our method whenever the interactions in $H_{\text {bilayer }}$ are constrained to satisfy at least one of the following three conditions: (i) $\mathcal{K}_{z}=\mathcal{J}_{z}$; (ii) $\mathcal{K}_{\perp}=\mathcal{J}_{\perp}$; (iii) $\mathcal{K}_{\perp}=-\mathcal{J}_{\perp}$. Fully frustrated bilayer systems [39-44], which have infinitely many conserved quantities, represent a special case with (i) and (ii) both being satisfied. The method also works when the $B$ sublattice only hosts a single spin-1/2 moment $\vec{S}_{r_{b}}$ that couples symmetrically to $\vec{S}_{\mathrm{I} r_{a}}$ and $\vec{S}_{\mathrm{II} r_{a}}$ on neighboring $A$ sublattice sites [45-47]:

$$
\begin{aligned}
H_{\text {mixed }}= & \sum_{\left\langle r_{a} r_{b}\right\rangle}\left(\mathcal{J}_{z} S_{\mathrm{I} r_{a}}^{z} S_{r_{b}}^{z}+\mathcal{J}_{\perp} \vec{S}_{\mathrm{I} r_{a}}^{\perp} \cdot \vec{S}_{r_{b}}^{\perp}+\mathrm{I} \leftrightarrow \mathrm{II}\right) \\
& +\sum_{r_{a}}\left(\mathcal{D}_{z} S_{\mathrm{I} r_{a}}^{z} S_{\mathrm{II} r_{a}}^{z}+\mathcal{D}_{\perp} \vec{S}_{\mathrm{I} r_{a}}^{\perp} \cdot \vec{S}_{\mathrm{II} r_{a}}^{\perp}\right) .
\end{aligned}
$$

For $\mathcal{D}_{\perp}>0$, the usual QMC method has a sign problem, which is no longer present in our QMC scheme. Our method is also expected to apply to other such models with infinitely many conserved quantities [48-54]. Additionally, (iii) includes interesting examples of frustrated bilayer magnets with full SU(2) symmetry and no extra conservation laws. Some of our results have been obtained independently in recent parallel work [55].

Key idea.-We use the stochastic series expansion (SSE) QMC framework [3] and work at each Bravais lattice site $r$ in the basis $\{|l, m\rangle\}$ of simultaneous eigenstates of the total spin $\vec{L}_{r}^{2}$ and its $z$ component $L_{r}^{z}$, with eigenvalues $l(l+1)$ and $m$, respectively. For $H_{\text {bilayer }}$, we define $\vec{L}_{r}=\vec{S}_{\mathrm{I} r}+\vec{S}_{\mathrm{II} r}$ on both sublattices. For $H_{\text {mixed }}$, this is modified on the $B$ sublattice by defining $\vec{L}_{r_{b}}=\vec{S}_{r_{b}}$. We decompose the Hamiltonian into terms living on bonds $\left\langle r_{a} r_{b}\right\rangle$ of the bipartite Bravais lattice, with the terms proportional to $\mathcal{D}_{z}$ and $\mathcal{D}_{\perp}$ at each site $r$ being shared equally among all bonds emanating from $r$ :

$$
\begin{aligned}
H_{1\left\langle r_{a} r_{b}\right\rangle}= & J_{z} L_{r_{a}}^{z} L_{r_{b}}^{z}+\zeta \Delta_{z}\left[\left(L_{r_{a}}^{z}\right)^{2}+\left(L_{r_{b}}^{z}\right)^{2}\right] \\
& +\zeta \Delta_{\perp}\left[\left(\vec{L}_{r_{a}}^{\perp}\right)^{2}+\left(\vec{L}_{r_{b}}^{\perp}\right)^{2}\right]-C, \\
H_{2\left\langle r_{a} r_{b}\right\rangle}^{ \pm}= & \frac{J_{\perp}}{2}\left(L_{r_{a}}^{ \pm} \cdot L_{r_{b}}^{\mp}\right) \\
H_{3\left\langle r_{a} r_{b}\right\rangle}= & K_{z} N_{r_{a}}^{z} N_{r_{b}}^{z}, \\
H_{4\left\langle r_{a} r_{b}\right\rangle}^{ \pm}= & \frac{K_{\perp}}{2}\left(N_{r_{a}}^{ \pm} \cdot N_{r_{b}}^{\mp}\right),
\end{aligned}
$$

with $\vec{L}_{r}^{\perp}$ the vector made of transverse (x/y) components of $\vec{L}_{r}, \vec{N}_{r}=\vec{S}_{\mathrm{I} r}-\vec{S}_{\mathrm{II} r}, L_{r}^{ \pm}=L_{r}^{x} \pm i L_{r}^{y}, N_{r}^{ \pm}=N_{r}^{x} \pm i N_{r}^{y}, \zeta$ the inverse coordination number of the bipartite lattice, $C$ a constant introduced to ensure negativity of all matrix elements of the diagonal operator $H_{1\left\langle r_{a} r_{b}\right\rangle}$ in our chosen basis, and $\Delta_{\mu}=\mathcal{D}_{\mu} / 2, J_{\mu}=\left(\mathcal{J}_{\mu}+\mathcal{K}_{\mu}\right) / 2, K_{\mu}=\left(\mathcal{J}_{\mu}-\right.$ $\left.\mathcal{K}_{\mu}\right) / 2$ (for $\mu=z, \perp$ ). Using this decomposition, we have $H_{\text {bilayer }}=\sum_{\left\langle r_{a} r_{b}\right\rangle} H_{1\left\langle r_{a} r_{b}\right\rangle}+H_{3\left\langle r_{a} r_{b}\right\rangle}+H_{2\left\langle r_{a} r_{b}\right\rangle}^{+}+H_{4\left\langle r_{a} r_{b}\right\rangle}^{+}+$ $H_{2\left\langle r_{a} r_{b}\right\rangle}^{-}+H_{4\left\langle r_{a} r_{b}\right\rangle}^{-} . H_{\text {mixed }}$, when decomposed in the same way, has analogs only of the $H_{1\left\langle r_{a} r_{b}\right\rangle}$ and $H_{2\left\langle r_{a} r_{b}\right\rangle}^{ \pm}$ terms [56].

Working within the SSE framework with this decomposition of $H_{\text {bilayer }}$, one writes

$$
\begin{aligned}
Z= & \sum_{n=0}^{\infty} \frac{1}{n ! T^{n}} \sum_{\mathcal{S}_{n}}\left\langle\alpha_{0}\left|\left(-H_{n}\right)\right| \alpha_{n-1}\right\rangle\left\langle\alpha_{n-1}\left|\left(-H_{n-1}\right)\right| \alpha_{n-2}\right\rangle \ldots \\
& \times\left\langle\alpha_{1}\left|\left(-H_{1}\right)\right| \alpha_{0}\right\rangle
\end{aligned}
$$

where the sum over operator strings $\mathcal{S}_{n}$ of length $n$ is implemented by allowing each $\left|\alpha_{j}\right\rangle$ to range over the full basis of states and each $H_{j}$ to range over all bond operators $H_{1\left\langle r_{a} r_{b}\right\rangle}, H_{2\left\langle r_{a} r_{b}\right\rangle}^{ \pm}, H_{3\left\langle r_{a} r_{b}\right\rangle}$, and $H_{4\left\langle r_{a} r_{b}\right\rangle}^{ \pm}$. Along with the factor of $1 /\left(n ! T^{n}\right)$, the product of matrix elements appearing in the summand serves as the Monte Carlo weight of each operator string, and the QMC simulation proceeds by performing an importance sampling of $Z$.

Proof of positive-weight property.-Clearly, the weight of an operator string does not depend on the choice of arbitrary phase factors attached to individual basis states, since these phase factors cancel in pairs in the product of matrix elements that sets the weight. Fixing these phases, we define the canonical cluster basis as follows: $|s\rangle=$ $\left(\left|\uparrow_{\text {I }} \downarrow_{\text {II }}\right\rangle-\left|\downarrow_{\text {I }} \uparrow_{\text {II }}\right\rangle\right) / \sqrt{2}, \quad\left|t_{+1}\right\rangle=\left|\uparrow_{\text {I }} \uparrow_{\text {III }}\right\rangle, \quad\left|t_{-1}\right\rangle=\left|\downarrow_{\text {I }} \downarrow_{\text {II }}\right\rangle$, and $\left|t_{0}\right\rangle=\left(\left|\uparrow_{\text {I }} \downarrow_{\text {II }}\right\rangle+\left|\downarrow_{\text {II }} \uparrow_{\text {II }}\right\rangle\right) / \sqrt{2}$. Next, we classify the off-diagonal matrix elements contributing to the weight of an operator string into five types (Fig. 1): (i) m-type processes that hop one quantum of $L^{z}$ along link $\left\langle r_{a} r_{b}\right\rangle$ between two neighboring triplet clusters, (ii) $l$-type processes that exchange the states $|s\rangle$ and $\left|t_{0}\right\rangle$ of two neighboring clusters, (iii) $p$-type processes that take neighboring singlet clusters and promote both to the $\left|t_{0}\right\rangle$ state or vice versa, (iv) $l m$-type processes that exchange singlet and triplet states of neighboring clusters and simultaneously hop one quantum of $L^{z}$, and (v) pm-type processes that take neighboring singlet clusters to states $\left|t_{ \pm 1}\right\rangle$ and $\left|t_{\mp 1}\right\rangle$, respectively, or vice versa.

All processes of a given type have a fixed sign for the corresponding matrix elements between basis states (Fig. 1). Therefore, a positive weight is guaranteed if $\mathcal{N}_{t}$, the number of occurrences (in any string $\mathcal{S}_{n}$ ) of $t$-type processes, has even parity for each type $t$. These parities are constrained by the periodicity of the operator string $\mathcal{S}_{n}$; i.e., the starting state $\left|\alpha_{0}\right\rangle$ is recovered after the action of $n$ operators. Since pair creation of the $l$ quantum number must be balanced by 
corresponding pair destruction processes, $\mathcal{N}_{p}+\mathcal{N}_{p m}$ must be even. Since a bipartite lattice has only loops of even length, the number of occurrences of processes that hop the $m$ quantum number must be even, implying that $\mathcal{N}_{m}+$ $\mathcal{N}_{p m}+\mathcal{N}_{l m}$ is even. By the same argument applied to the $l$ quantum number, $\mathcal{N}_{l}+\mathcal{N}_{l m}$ must also be even.

Since $H_{2\left\langle r_{a} r_{b}\right\rangle}^{ \pm}$gives rise only to $m$-type processes, while $H_{3\left\langle r_{a} r_{b}\right\rangle}$ gives rise to $l$-type and $p$-type processes and $H_{4\left\langle r_{a} r_{b}\right\rangle}^{ \pm}$gives rise to $l m$-type and $p m$-type processes, $K_{\perp}=$ $0\left(K_{z}=0\right)$ implies $\mathcal{N}_{l m}=\mathcal{N}_{p m}=0\left(\mathcal{N}_{l}=\mathcal{N}_{p}=0\right)$. The periodicity constraints then imply that all nonzero $\mathcal{N}_{t}$ are even in both these cases. As a result, in both these cases, each $\mathcal{S}_{n}$ has positive weight in this QMC scheme regardless of the sign of all nonzero couplings. On the other hand, if $J_{\perp}=0$, i.e., $\mathcal{N}_{m}=0$, only $p m$-type processes can create or destroy pairs of $m= \pm 1$ states on neighboring sites, thus ensuring that $\mathcal{N}_{p m}$ is even. Along with the other periodicity constraints, this again implies that all nonzero $\mathcal{N}_{t}$ are even, yielding a sign-problem-free method whenever $J_{\perp}=0$, independent of the sign of other couplings.

Thus, the Monte Carlo weight is positive for frustrated bilayer magnets with Hamiltonian $H_{\text {bilayer }}$ when at least one of the following conditions is satisfied: (i) $\mathcal{K}_{z}=\mathcal{J}_{z}$; (ii) $\mathcal{K}_{\perp}=\mathcal{J}_{\perp}$; (iii) $\mathcal{K}_{\perp}=-\mathcal{J}_{\perp}$. When (i) and (ii) are both satisfied, one obtains fully frustrated bilayer systems [39-44] with infinitely many conservation laws, which can have either SU(2) or U(1) symmetry. Additionally, (iii) also contains other examples of $\mathrm{SU}(2)$ symmetric frustrated magnets (e.g., for $J_{z}=J_{\perp}=0, K_{z}=K_{\perp}>0$, and $\left.\Delta_{z}=\Delta_{\perp}<0\right)$ and no extra conservation laws. A similar argument establishes the absence of a sign problem for $H_{\text {mixed }}$ [56].

Alternately, this positive-weight property can be made explicit by switching from the canonical cluster basis to a rotated basis obtained by attaching phase factors $e^{i \theta_{|m\rangle}+i \eta_{|l m\rangle}}$ to the states $|l, m\rangle$. Here, the $\theta_{|l m\rangle}$ are $r$ independent, while the $\eta_{|l, m\rangle}$ are 0 on the $B$ sublattice and constant on the $A$ sublattice. These phases are chosen in each of these three generic cases to ensure that every nonzero contribution to the weight in the rotated basis is explicitly positive. For instance, when $K_{\perp}=0$, we set $\eta_{\left|t_{ \pm}\right\rangle}=\theta_{|l, m\rangle}=0$ (for all $l, m$ ) while independently choosing $\eta_{|s\rangle}$ to be 0 or $\pi$ and $\eta_{\left|t_{0}\right\rangle}$ to be 0 or $\pi$ depending on the signs of $K_{z}$ and $J_{\perp}$. The other sign-free cases can be handled with slightly different choices for these phase factors [56]. The positive-weight property of $H_{\text {mixed }}$ can also be made explicit in the same way [56].

Implementation.-A key advantage of this QMC approach is that the usual SSE framework [3] remains valid with no change in the construction of the diagonal update and one new feature in the construction of directed loop updates: Three different kinds of directed loop updates $[57,58]$ are now possible, involving changes to the $m$ quantum number, the $l$ quantum number, or both. Additionally, to improve statistics, one can use parallel

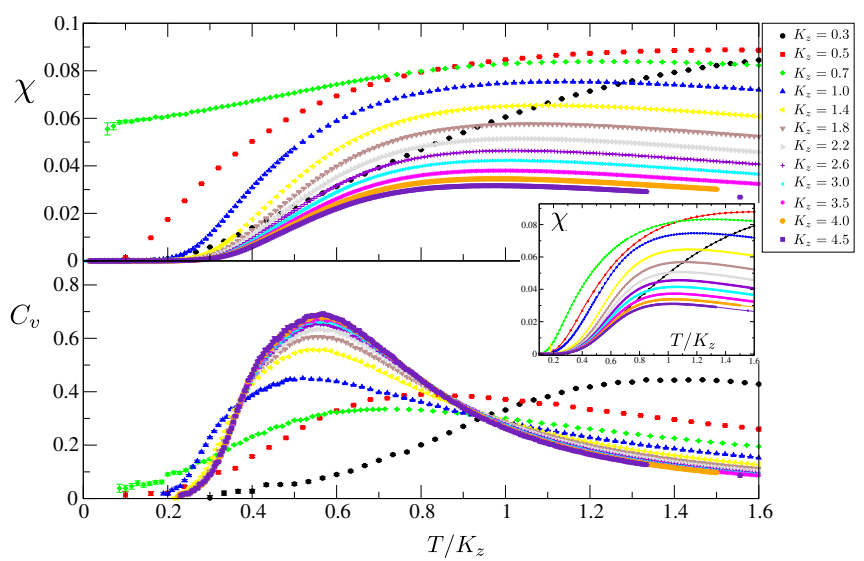

FIG. 2. Temperature $(T)$ dependence of the susceptibility and specific heat of $H_{\text {bilayer }}$ with $\mathcal{D}_{z}=\mathcal{D}_{\perp}=1, \mathcal{J}_{\perp}=\mathcal{K}_{\perp}=1$, $\mathcal{J}_{z}=1+K_{z}$, and $\mathcal{K}_{z}=1-K_{z}$. Symbols display data for a sample with $L=64$ unit cells, plotted for a variety of values of $K_{z}$. The inset shows the perfect agreement between QMC data (symbols) and exact diagonalization results (lines) for $L=6$.

tempering [59] as well as an additional local update, which identifies worldlines that are only touched by diagonal vertices, and changes their state using Metropolis-type acceptance probabilities.

Benchmarks.-Our implementation, which incorporates all these updates, has been successfully benchmarked against exact diagonalization in spatial dimension $d=1$ for all the sign-free cases, including the two special cases with infinitely many conserved quantities [56]. In Fig. 2 (inset), we illustrate this for a representative example, focusing on the susceptibility $\chi=\beta\left\langle\left(S^{z}\right)^{2}\right\rangle / N$ and specific heat per spin $C_{v}=\frac{1}{N}(d\langle H\rangle / d T)$ for $H_{\text {bilayer }}$ in $d=1$, with $K_{\perp}=0(N=2 L$ is the number of spins $1 / 2$ in a ladder of length $L$ ). Data in the main panel illustrate the power of the method, which allows us to access the thermodynamics of this frustrated ladder for fairly large $L$ up to low $T$ and for a range of values of $K_{z}$.

Numerical results in $d=2$.-We now consider $H_{\text {bilayer }}$ on a square Bravais lattice in the presence of an additional magnetic field, which our method can handle without a sign problem: $H=H_{\text {bilayer }}-h \sum_{r}\left(S_{\mathrm{I} r}^{z}+S_{\mathrm{II} r}^{z}\right)$ (the magnetic field modifies only weights of the diagonal vertices). The physics of the fully frustrated special case $\left(K_{z}=K_{\perp}=0\right)$ in a certain field range was argued [60] to be dominated at low temperatures by sublattice-ordered configurations in which two-spin clusters on one spontaneously chosen sublattice are in the triplet state $\left|t_{+1}\right\rangle$, while two-spin clusters on the other sublattice are in a singlet state $|s\rangle$, allowing a low-temperature mapping to the ordered phase of hard squares on the square lattice. This predicts that the system undergoes a temperature-driven phase transition in the $2 \mathrm{~d}$ Ising universality class to a high-temperature phase in which sublattice symmetry is restored $[60,61]$. 

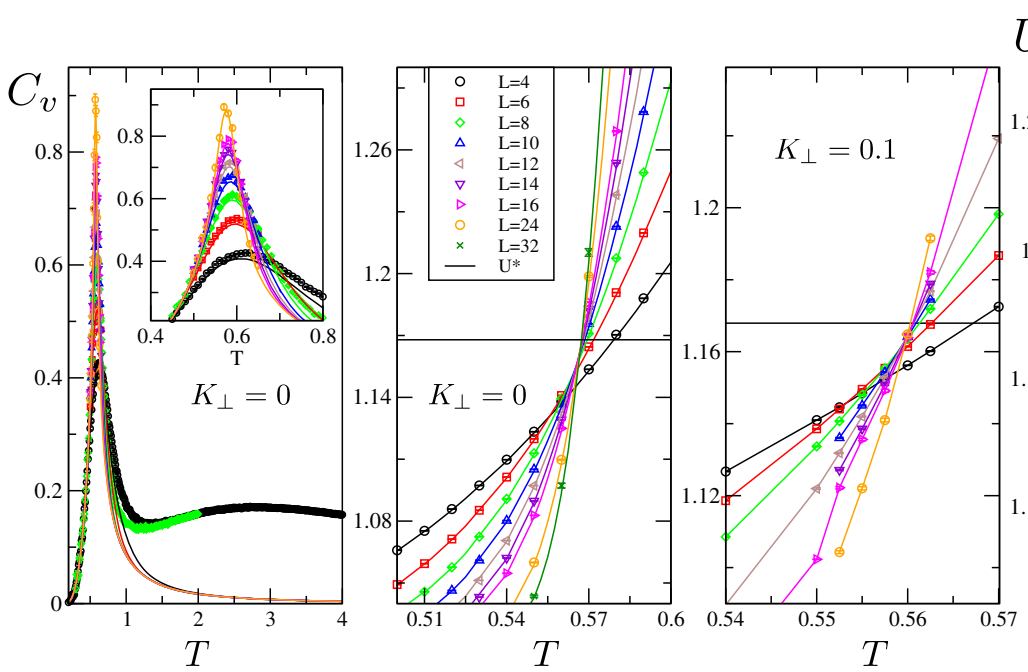

$U$

FIG. 3. QMC results (symbols) for $H_{\text {bilayer }}$ in a field on the square lattice, with $\mathcal{J}_{z}=\mathcal{K}_{z}=1, \mathcal{D}_{z}=\mathcal{D}_{\perp}=5, \mathcal{J}_{\perp}=1+K_{\perp}$, $\mathcal{K}_{\perp}=1-K_{\perp}$, and $h=7$. Left panel: Specific heat $C_{v}$ for $K_{\perp}=0$ (inset zooms into the critical range). Right panels: Binder cumulant $U=\left\langle m_{s}^{4}\right\rangle /\left\langle m_{s}^{2}\right\rangle^{2}$ of the staggered magnetization $m_{s}=\sum_{r}(-)^{r}\left(S_{\mathrm{I} r}^{z}+S_{\mathrm{II} r}^{z}\right)$. The critical temperature $T_{c}$, estimated by the crossing point of $U$, decreases with $K_{\perp} . U$ at the estimated $T_{c}$ tends to the $2 \mathrm{~d}$ Ising critical value $U^{*}=1.16793$ [62] at large $L$ for all $K_{\perp}$ displayed. The solid lines in the $K_{\perp}>0$ panels are guides to the eye. At $K_{\perp}=0$, they denote results for the $2 \mathrm{~d}$ classical Ising model at $T_{\text {Ising }}=4 T$.

We have performed a high-precision QMC test of this prediction using a finite-size scaling analysis for samples with up to $N=2 L^{2}=2048$ spins $1 / 2$, both for the fully frustrated special case and in the presence of nonzero $K_{\perp}$ (previous quantum simulations were limited to $K_{\perp}=0$ and $N=20$ ). Our determination of the critical value of the Binder cumulant of the staggered magnetization provides clear evidence that this transition indeed belongs to the $2 \mathrm{~d}$ Ising universality class both for $K_{\perp}=0$ and for nonzero $K_{\perp}$ (albeit with stronger finite-size effects in this case). Indeed, our QMC data for the specific heat and the Binder cumulant close to the phase transition (Fig. 3) are almost identical to those of the classical $2 \mathrm{~d}$ Ising model when $K_{\perp}=0$ but deviate from the classical results outside the critical region, underscoring the nontrivial nature of this correspondence. These deviations become much more significant for nonzero $K_{\perp}$. Our method thus enables an investigation of the full parameter regime, including where the hard-square mapping breaks down, both in the fully frustrated special case and when $K_{\perp} \neq 0$ (the effect of $K_{z} \neq 0$ can also be studied).

Discussion.-Clearly, the method presented here can be applied to a large class of frustrated magnets [60,63-67] and models closely related to specific strongly correlated materials: For instance, a generalized version of $H_{\text {mixed }}$ has been argued to be a good model for the mineral azurite $\mathrm{Cu}_{3}\left(\mathrm{CO}_{3}\right)_{2}(\mathrm{OH})_{2}$ [47], and the specific heat of the fully frustrated ladder [56] has similar features with the Shastry-Sutherland compound $\mathrm{SrCu}_{2}\left(\mathrm{BO}_{3}\right)_{2}$ [55]. This QMC method also enables the search for finite- $T$ signatures of multitriplet bond states, as shown in Ref. [55]. Additionally, it offers the possibility of using large-scale unbiased simulations to study interesting quantum phase transitions driven by the competition between different exchange interactions. As we illustrated, a magnetic field (in the $z$ direction) can also be included, thus allowing one to study magnetization processes and plateaux of such frustrated magnets $[41,44,52,54]$. On the flip side, we note that this QMC scheme does not remain sign-free when $J_{\perp}, K_{\perp}$, and $K_{z}$ are all nonzero in the general bilayer Hamiltonian $H_{\text {bilayer }}$. The simple sequence of processes shown in Fig. 4 for a single plaquette of a square lattice provides an explicit illustration of this limitation.

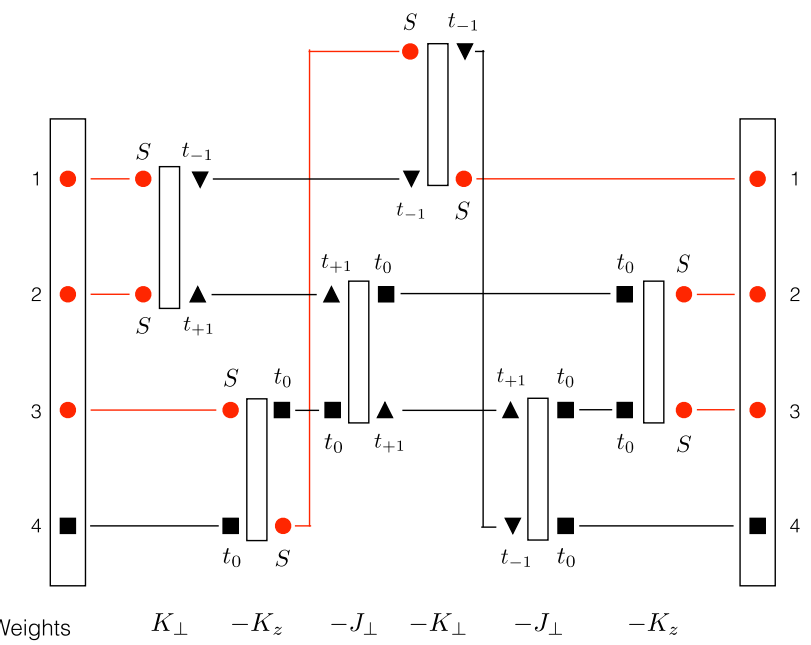

FIG. 4. This operator string for a single plaquette of the Bravais lattice of the bilayer system illustrates the origin of the sign problem faced when simulating the general bilayer Hamiltonian $H_{\text {bilayer }}$ : Its weight is negative, independent of the signs of the nonzero couplings $J_{\perp}, K_{z}$, and $K_{\perp}$. 
Nevertheless, this construction of negative-weight configurations relies on the existence of loops in the underlying bipartite Bravais lattice and leaves open the possibility that this sign problem could be controlled in 1d systems with open boundaries. In summary, our work has led to a solution of the sign problem for a large and interesting class of frustrated quantum magnets. Given the ubiquity of the sign problem in computational physics, we hope that the strategy outlined in this work can be adapted to other systems.

We thank S. Wessel for generously sharing with us some details of the recent parallel work Ref. [55] and for stimulating discussions regarding the sign problem in the general case. We gratefully acknowledge J. Richter for suggesting the study of the finite-temperature Ising transition in the square bilayer system in a field. One of us (K. D.) also gratefully acknowledges the hospitality of ECT* (Trento), ICTP (Trieste), and ICTS-TIFR (Bengaluru) during the writing of this Letter. This work was supported by the Indo-French Centre for the Promotion of Advanced Research (IFCPAR/CEFIPRA Project 4504-1) and performed using numerical resources from GENCI (Grants No. 2014x2014050225 and No. 2015-x2015050225) and CALMIP. Our QMC code is based on the SSE code of the ALPS libraries $[58,68]$.

[1] Strongly Correlated Systems Numerical Methods, edited by A. Avella and F. Mancini, Springer Series in Solid State Sciences Vol. 176 (Springer, Berlin, 2013).

[2] W. Krauth, Algorithms and Computations, Oxford Master Series in Statistical Computational and Theoretical Physics (Oxford University, New York, 2006).

[3] A. W. Sandvik, Computational studies of quantum spin systems, AIP Conf. Proc. 1297, 135 (2010).

[4] D. C. Johnston, R. K. Kremer, M. Troyer, X. Wang, A. Klümper, S. L. Bud'ko, A. F. Panchula, and P. C. Canfield, Thermodynamics of spin $\mathrm{S}=1 / 2$ antiferromagnetic uniform and alternating-exchange Heisenberg chains, Phys. Rev. B 61, 9558 (2000).

[5] Introduction to Frustrated Magnetism Materials, Experiments, Theory, edited by C. Lacroix, P. Mendels, and F. Mila, Springer Series in Solid State Sciences Vol. 176 (Springer, Berlin, 2011).

[6] S. Wessel, in Lecture Notes of the Autumn School Correlated Electrons 2013, edited by E. Pavarini, E. Koch, and U. Schollwck (Forschungszentrum, Julich, 2013).

[7] R. K. Kaul, R. G. Melko, and A. W. Sandvik, Bridging lattice-scale physics and continuum field theory with quantum Monte Carlo simulations, Annu. Rev. Condens. Matter Phys. 4, 179 (2013).

[8] M. Troyer and U.-J. Wiese, Computational Complexity and Fundamental Limitations to Fermionic Quantum Monte Carlo Simulations, Phys. Rev. Lett. 94, 170201 (2005).

[9] R. Moessner and S. L. Sondhi, Ising models of quantum frustration, Phys. Rev. B 63, 224401 (2001).
[10] S. V. Isakov and R. Moessner, Interplay of quantum and thermal fluctuations in a frustrated magnet, Phys. Rev. B 68, 104409 (2003).

[11] S. Wessel and M. Troyer, Supersolid Hard-Core Bosons on the Triangular Lattice, Phys. Rev. Lett. 95, 127205 (2005).

[12] D. Heidarian and K. Damle, Persistent Supersolid Phase of Hard-Core Bosons on the Triangular Lattice, Phys. Rev. Lett. 95, 127206 (2005).

[13] R. G. Melko, A. Paramekanti, A. A. Burkov, A. Vishwanath, D. N. Sheng, and L. Balents, Supersolid Order from Disorder: Hard-Core Bosons on the Triangular Lattice, Phys. Rev. Lett. 95, 127207 (2005).

[14] S. V. Isakov, S. Wessel, R. G. Melko, K. Sengupta, and Y. B. Kim, Hard-Core Bosons on the Kagome Lattice: ValenceBond Solids and Their Quantum Melting, Phys. Rev. Lett. 97, 147202 (2006).

[15] S. V. Isakov, Y. B. Kim, and A. Paramekanti, Spin-Liquid Phase in a Spin-1/2 Quantum Magnet on the Kagome Lattice, Phys. Rev. Lett. 97, 207204 (2006).

[16] A. Banerjee, S. V. Isakov, K. Damle, and Y. B. Kim, Unusual Liquid State of Hard-Core Bosons on the Pyrochlore Lattice, Phys. Rev. Lett. 100, 047208 (2008).

[17] A. Sen, K. Damle, and T. Senthil, Superfluid insulator transitions of hard-core bosons on the checkerboard lattice, Phys. Rev. B 76, 235107 (2007).

[18] K. Damle and T. Senthil, Spin Nematics and Magnetization Plateau Transition in Anisotropic Kagome Magnets, Phys. Rev. Lett. 97, 067202 (2006).

[19] A. Sen, F. Wang, K. Damle, and R. Moessner, Triangular and Kagome Antiferromagnets with a Strong Easy-Axis Anisotropy, Phys. Rev. Lett. 102, 227001 (2009).

[20] A. Sen, K. Damle, and A. Vishwanath, Magnetization Plateaus and Sublattice Ordering in Easy-Axis Kagome Lattice Antiferromagnets, Phys. Rev. Lett. 100, 097202 (2008).

[21] E. Berg, M. A. Metlitski, and S. Sachdev, Sign-problem-free quantum Monte Carlo of the onset of antiferromagnetism in metals, Science 338, 1606 (2012).

[22] Y.-P. Huang, G. Chen, and M. Hermele, Quantum Spin Ices and Topological Phases from Dipolar-Octupolar Doublets on the Pyrochlore Lattice, Phys. Rev. Lett. 112, 167203 (2014).

[23] F. F. Assaad and H. G. Evertz, in Proceedings of the Heraeus Summer School, Greifswald, Springer Lecture Notes in Physics 739, edited by H. Fehske et al. (Springer, New York, 2008).

[24] C. Wu and S.-C. Zhang, Sufficient condition for absence of the sign problem in the fermionic quantum Monte Carlo algorithm, Phys. Rev. B 71, 155115 (2005).

[25] L. Messio and F. Mila, Entropy Dependence of Correlations in One-Dimensional SU(N) Antiferromagnets, Phys. Rev. Lett. 109, 205306 (2012).

[26] E. F. Huffman and S. Chandrasekharan, Solution to sign problems in half-filled spin-polarized electronic systems, Phys. Rev. B 89, 111101 (2014).

[27] L. Wang, Y.-H. Liu, M. Iazzi, M. Troyer, and G. Harcos, Split Orthogonal Group: A Guiding Principle for SignProblem-Free Fermionic Simulations, Phys. Rev. Lett. 115, 250601 (2015). 
[28] Z.-X. Li, Y.-F. Jiang, and H. Yao, Solving the fermion sign problem in quantum Monte Carlo simulations by Majorana representation, Phys. Rev. B 91, 241117(R) (2015).

[29] Z. C. Wei, C. Wu, Y. Li, S. Zhang, and T. Xiang, Majorana Positivity and the Fermion Sign Problem of Quantum Monte Carlo Simulations, Phys. Rev. Lett. 116, 250601 (2016).

[30] P. Henelius and A. W. Sandvik, Sign problem in Monte Carlo simulations of frustrated quantum spin systems, Phys. Rev. B 62, 1102 (2000).

[31] W. Bietenholz, A. Pochinsky, and U.-J. Wiese, Meron Cluster Simulation of the Theta Vacuum in the 2-d O(3) Model, Phys. Rev. Lett. 75, 4524 (1995).

[32] S. Chandrasekharan and U.-J. Wiese, Meron-Cluster Solution of Fermion Sign Problems, Phys. Rev. Lett. 83, 3116 (1999).

[33] M. Nyfeler, F.-J. Jiang, F. Kämpfer, and U.-J. Wiese, Nested Cluster Algorithm for Frustrated Quantum Antiferromagnets, Phys. Rev. Lett. 100, 247206 (2008).

[34] T. Nakamura, Vanishing of the negative-sign problem of quantum Monte Carlo simulations in one-dimensional frustrated spin systems, Phys. Rev. B 57, R3197 (1998).

[35] K. Okunishi and K. Harada, Symmetry-protected topological order and negative-sign problem for $\mathrm{SO}(\mathrm{N})$ bilinearbiquadratic chains, Phys. Rev. B 89, 134422 (2014).

[36] S. D. Geraedts and O. I. Motrunich, Model of fractionalization of Faraday lines in compact electrodynamics, Phys. Rev. B 90, 214505 (2014).

[37] S. D. Geraedts and O. I. Motrunich, Model Realization and Numerical Studies of a Three-Dimensional Bosonic Topological Insulator and Symmetry-Enriched Topological Phases, Phys. Rev. X 4, 041049 (2014).

[38] S. D. Geraedts and O. I. Motrunich, Exact realization of integer and fractional quantum Hall phases in $\mathrm{U}(1) \times \mathrm{U}(1)$ models in $(2+1)$ d, Ann. Phys. (Amsterdam) 334, 288 (2013).

[39] M. P. Gelfand, Linked-tetrahedra spin chain: Exact ground state and excitations, Phys. Rev. B 43, 8644 (1991).

[40] A. K. Kolezhuk and H.-J. Mikeska, Finitely correlated generalized spin ladders, Int. J. Mod. Phys. B 12, 2325 (1998).

[41] A. Honecker, F. Mila, and M. Troyer, Magnetization plateaux and jumps in a class of frustrated ladders: A simple route to a complex behaviour, Eur. Phys. J. B 15, 227 (2000).

[42] W. Brenig and K. W. Becker, Magnetism of a tetrahedral cluster spin chain, Phys. Rev. B 64, 214413 (2001).

[43] E. Chattopadhyay and I. Bose, Magnetization of coupled spin clusters in ladder geometry, Phys. Rev. B 65, 134425 (2002).

[44] K. Totsuka and H.-J. Miseka, Low-lying excitations and magnetization process of coupled tetrahedral systems, Phys. Rev. B 66, 054435 (2002).

[45] K. Takano, K. Kubo, and H. Sakamoto, Ground states with cluster structures in a frustrated Heisenberg chain, J. Phys. Condens. Matter 8, 6405 (1996).

[46] H. Niggemann, G. Uimin, and J. Zittartz, Mixed Heisenberg chains: I. The ground-state problem, J. Phys. Condens. Matter 9, 9031 (1997).
[47] A. Honecker, S. Hu, R. Peters, and J. Richter, Dynamic and thermodynamic properties of the generalised diamond chain model for azurite, J. Phys. Condens. Matter 23, 164211 (2011).

[48] N. B. Ivanov and J. Richter, Competition between plaquette and dimer phases in Heisenberg chains, Phys. Lett. A 232, 308 (1997).

[49] J. Richter, N. B. Ivanov, and J. Schulenburg, The antiferromagnetic spin-1/2 chain with competing dimers and plaquettes: Numerical versus exact results, J. Phys. Condens. Matter 10, 3635 (1998).

[50] A. Koga, K. Okunishi, and N. Kawakami, First-order quantum phase transition in the orthogonal-dimer spin chain, Phys. Rev. B 62, 5558 (2000).

[51] C. Gros, R. Valentí, J. V. Alvarez, K. Hamacher, and W. Wenzel, Test of the frustrated spin-cluster model to describe the low-temperature physics of $\mathrm{NaV}_{2} \mathrm{O}_{5}$, Phys. Rev. B 62 , R14617 (2000).

[52] J. Schulenburg and J. Richter, Infinite series of magnetization plateaus in the frustrated dimer-plaquette chain, Phys. Rev. B 65, 054420 (2002).

[53] A. Honecker and W. Brenig, Magnetic properties of a spin-1/2 quadrumer chain, Phys. Rev. B 63, 144416 (2001).

[54] S. R. Manmana, J.-D. Picon, K. P. Schmidt, and F. Mila, Unconventional magnetization plateaus in a ShastrySutherland spin tube, Europhys. Lett. 94, 67004 (2011).

[55] A. Honecker, S. Wessel, R. Kerkdyk, T. Pruschke, F. Mila, and B. Normand, Thermodynamic properties of highly frustrated quantum spin ladders: Influence of many-particle bound states, Phys. Rev. B 93, 054408 (2016).

[56] See Supplemental Material at http://link.aps.org/ supplemental/10.1103/PhysRevLett.117.197203 for details of the QMC simulation of $H_{\text {mixed }}$ (including a proof of positive-weight), and results on one-dimensional systems which have been used to benchmark the method.

[57] O. F. Syljuåsen and A. W. Sandvik, Quantum Monte Carlo with directed loops, Phys. Rev. E 66, 046701 (2002).

[58] F. Alet, S. Wessel, and M. Troyer, Generalized directed loop method for quantum Monte Carlo simulations, Phys. Rev. E 71, 036706 (2005).

[59] K. Hukushima and K. Nemoto, Exchange Monte Carlo method and application to spin glass simulations, J. Phys. Soc. Jpn. 65, 1604 (1996).

[60] J. Richter, O. Derzhko, and T. Krokhmalskii, Finitetemperature order-disorder phase transition in a frustrated bilayer quantum Heisenberg antiferromagnet in strong magnetic fields, Phys. Rev. B 74, 144430 (2006).

[61] O. Derzhko, T. Krokhmalskii, and J. Richter, Emergent Ising degrees of freedom in frustrated two-leg ladder and bilayer $s=1 / 2$ Heisenberg antiferromagnets, Phys. Rev. B 82, 214412 (2010).

[62] G. Kamieniarz and H. W. J. Blöte, Universal ratio of magnetization moments in two-dimensional Ising models, J. Phys. A 26, 201 (1993).

[63] E. Müller-Hartmann, R. R. P. Singh, C. Knetter, and G. S. Uhrig, Exact Demonstration of Magnetization Pleateaus and First Order Dimer-Néel Phase Transitions in a Modified 
Shastry-Sutherland Model for $\mathrm{SrCu}_{2}\left(\mathrm{BO}_{3}\right)_{2}$, Phys. Rev. Lett. 84, 1808 (2000).

[64] H. Q. Lin, J. L. Shen, and H. Y. Shik, Exactly soluble quantum spin models on a double layer: The net spin model, Phys. Rev. B 66, 184402 (2002).

[65] J. Rössler and D. Gottlieb, Analysis of a family of Heisenberg systems with simple eigenfunctions for the ground state and low lying excitations, Phys. Rev. B 72, 024443 (2005).
[66] M. Mambrini, J. Trébosc, and F. Mila, Residual entropy and spin gap in a one-dimensional frustrated antiferromagnet, Phys. Rev. B 59, 13806 (1999).

[67] O. Rojas and F. C. Alcaraz, Phase diagram of a coupled tetrahedral Heisenberg model, Phys. Rev. B 67, 174401 (2003).

[68] A. F. Albuquerque et al., The ALPS project release 1.3: Open source software for strongly correlated systems, J. Magn. Magn. Mater. 310, 1187 (2007). 